\title{
HARMONIC ANALYSIS OF LOCAL TIMES AND SAMPLE FUNCTIONS OF GAUSSIAN PROCESSES $\left({ }^{1}\right)$
}

\author{
BY \\ SIMEON M. BERMAN
}

Introduction and summary. Let $I$ be a topological space, $\mathscr{B}$ its class of Borel sets, $\mu$ a positive finite measure on $\mathscr{B}$, and $x=x(t), t \in I$, a real valued Borel function. Define the measure

$$
\nu(A)=\mu\left(x^{-1}(A)\right)
$$

on the linear Borel sets $A$. If $\nu$ is absolutely continuous with respect to the usual linear Borel measure, then its derivative is called the local time of $x$. In this paper we deduce some properties of the variation of $x$ from smoothness properties of the local time when $\mu$ is fixed and $x$ is a sample function of a Gaussian process. The main results are Theorems 5.1 and 5.2 which imply: If $X(t), 0 \leqq t \leqq 1$, is a Gaussian process with mean 0 satisfying $E(X(s)-X(t))^{2} \geqq C|t-s|^{\beta}$, for some $\beta, 0<\beta<2$, then the sample functions

(a) are of unbounded $\gamma$-variation for $\gamma<2 / \beta$;

(b) nowhere satisfy a Hölder condition of order $2 /(2 m+\varepsilon+1)$ if $\beta<2 /(2 m+\varepsilon+1)$, where $m$ is a nonnegative integer and $0 \leqq \varepsilon<1$;

(c) are nowhere differentiable if $\beta<1$;

(d) return infinitely often to every neighborhood of almost all the points they visit.

The result (c) was stated without proof in [5] for a stationary process whose covariance function $r$ is not twice differentiable at the origin. A related result was proved by Yeh [7] under the additional assumption that $X(\cdot)$ is continuous. The paper of Marcus [4] contains results on Hölder conditions for processes with stationary increments satisfying the condition that $E(X(t)-X(0))^{2}$ is concave in some neighborhood of 0 , or related conditions.

Using a previous result on analytic local times [2], we prove in $\S 6$ that Lévy's Brownian motion over Hilbert space has sample functions unbounded over certain compact ellipsoids (cf. [1]).

1. Square integrable local times. Put

$$
f(u)=\int_{-\infty}^{\infty} e^{i u x} d \nu([-\infty, x]), \quad-\infty<u<\infty
$$

Received by the editors December 19, 1968.

(1) This paper represents results obtained at the Courant Institute of Mathematical Sciences, New York University, under the sponsorship of the National Science Foundation Grant NSF-GP-7378 and NSF-GW-2049. 
then $f$ is representable as

$$
f(u)=\int_{I} \exp (\operatorname{iux}(t)) d \mu(t)
$$

because $x=x(t)$ is a measure-preserving transformation on $(I, \mu)$ to $(R, \nu)$. If $f$ is square integrable, then $\nu$ is absolutely continuous and the local time is square integrable (cf. [2]). We denote the latter by $\phi(x)$. For every square integrable Borel function $g$ we have

$$
\int_{I} g(x(t)) d \mu(t)=\int_{-\infty}^{\infty} g(x) \phi(x) d x .
$$

Now we introduce the relative local time $\phi_{B}$, where $B$ is a Borel subset of $I$ : it is the local time of the restriction of $x$ to $B$. It is a consequence of the definition of the Radon-Nikodym derivative that $\phi_{B}(x) \leqq \phi(x)$ for almost all $x$; hence, $\phi_{B}$ is square integrable if $\phi$ is. If $\phi$ satisfies the equation (1.2), then $\phi_{B}$ satisfies a similar equation with $B$ in place of $I$ as the domain of integration. As a derivative with respect to Borel measure, the local time is Borel measurable; thus, from the definition of the relative local time it follows that if $\phi$ is square integrable, then, by

(1.3) $\int_{C} \phi_{B}(x(t)) d \mu(t)=\int_{-\infty}^{\infty} \phi_{B}(x) \phi_{C}(x) d x=\int_{B} \phi_{C}(x(t)) d \mu(t), \quad B, C, \varepsilon, \mathscr{B}$.

If $\mu(B \cap C)=0$, then

$$
\phi_{B \cup C}=\phi_{B}+\phi_{C}
$$

Lemma 1.1. If $\phi$ is square integrable, then $\phi(x(t))>0$, for almost all $t$.

Proof. Let $B$ be the set of $t$ upon which $\phi(x(t))=0$; then, from (1.3) with $C=I$, we have

$$
0=\int_{B} \phi(x(t)) d \mu(t)=\int_{-\infty}^{\infty} \phi(x) \phi_{B}(x) d x .
$$

Since $\phi$ dominates $\phi_{B}$, the last integral dominates $\int_{-\infty}^{\infty} \phi_{B}^{2}(x) d x$; thus $\phi_{B}$ vanishes almost everywhere, and so

$$
\mu(B)=\int_{-\infty}^{\infty} \phi_{B}(x) d x=0
$$

2. Recurrence properties of a class of functions with square integrable local times. In this section we take $I$ to be the closed unit interval and $\mu$ the usual Borel measure (Lebesgue measure on the Borel sets). Let $x(t), 0 \leqq t \leqq 1$, be a Borel function with a square integrable local time. For each positive integer $n$, Let $I_{n k}$ be the closed interval $\left[2^{-n}(k-1), 2^{-n} k\right], k=1, \ldots, 2^{n}$. Let $\phi_{n k}$ be the local time relative to $I_{n k}$, and $f_{n k}$ the corresponding Fourier transform. 
LEMMA 2.1. The sequence of sums

$$
\sum_{k=1}^{2^{n}} \int_{-\infty}^{\infty}\left|f_{n k}(u)\right|^{2} d u, \quad n=1,2, \ldots
$$

is nonincreasing; consequently, it converges.

Proof. Each interval $I_{n k}$ is the union of two intervals $I_{n+1, j}$ and $I_{n+1, j+1}$. Their intersection contains one point so that, by (1.4):

$$
\phi_{n k}=\phi_{n+1, j}+\phi_{n+1, j+1}
$$

moreover $\left(\phi_{n+1, j}+\phi_{n+1, j+1}\right)^{2} \geqq \phi_{n+1, j}^{2}+\phi_{n+1, j+1}^{2}$ because the local time is nonnegative; therefore, $\phi_{n k}^{2} \geqq \phi_{n+1, j}^{2}+\phi_{n+1, j+1}^{2}$. This inequality is preserved under integration; therefore, by Parseval's theorem, the same inequality also holds for the integrals of the squared moduli of the corresponding Fourier transforms. This completes the proof.

For each real number $y$, let $N(y)$ be the number (finite or infinite) of elements in the set

$\{t: 0 \leqq t \leqq 1, y$ is in the closure of the image of every neighborhood of $t\}$.

(If the function is continuous $N(y)$ is the number of times that it assumes the value $y$.) Let $N_{n}(y)$ be the number of positive elements in the set $\phi_{n k}(y), k=1, \ldots, 2^{n}$, $n=1,2, \ldots$

LEMMA 2.2. For each $y$, the sequence $\left\{N_{n}(y)\right\}$ is nondecreasing; thus, it has a limit which may be finite or infinite.

Proof. The equation (2.2) implies that for every positive element of the set $\left\{\phi_{n k}(y), k=1, \ldots, 2^{n}\right\}$ there is at least one positive element in the set $\left\{\phi_{n+1, k}(y)\right.$, $\left.k=1, \ldots, 2^{n+1}\right\}$.

LEMMA 2.3. If $N_{n}(y) \rightarrow \infty$ for $n \rightarrow \infty$, then $N(y)=\infty$.

Proof. Without losing generality, we may suppose that the local time vanishes outside the closure of the range of $x$; the reasoning is as follows. Let $y$ be a point outside the closure of the range; then some neighborhood of $y$ is never visited by the function $x$; thus, the integral of the local time over the neighborhood is 0 ; therefore, the local time vanishes almost everywhere in this neighborhood. Recall that the Radon-Nikodym derivative is unique in its definition except for a Borel set of measure 0 ; thus, the local time may, if necessary, be redefined so as to vanish everywhere in this neighborhood of $y$. Since the complement of the closure of the range may be covered by countably many such neighborhoods, the derivative may be so redefined on each of these, each time on a set of measure 0 ; thus, the local time, by an alteration on a set of measure 0 , may be taken to vanish throughout the complement of the closure of the range. 
Under the hypothesis of the lemma, for every positive integer $M$, there is an index $n$ sufficiently large so that $N_{n}(y)>2 M$. The assertion of the previous paragraph implies that $y$ belongs to the closure of the range of $x(t), t \in I_{n k}$, for more than $2 M$ indices $k$; thus, $N(y)>M$. Since $M$ is arbitrary, the lemma is proved.

Lemma 2.4. The set $\{y: N(y)<\infty\}$ is Borel measurable.

Proof. For $\varepsilon, 0<\varepsilon<1$, form a finite covering of $[0,1]$ by open intervals $J$ of length less than $\varepsilon$ such that each point is covered by at most two intervals $J$. For each $y$, let $N_{\varepsilon}(y)$ be the number of intervals $J$ whose images have closures containing $y$; $N_{\varepsilon}(y)$ is a Borel function because $x$ is. $N(y)$ is infinite if and only if $N_{\varepsilon}(y)$ becomes infinite for $\varepsilon \rightarrow 0$; indeed, if $t$ is a point whose every neighborhood has an image with closure containing $y$, then there are one or two intervals $J$ whose images also have closures containing $y$. This proves:

$$
\{y: N(y)<\infty\}=\left\{y: \liminf _{\varepsilon \rightarrow 0} N_{\varepsilon}(y)<\infty\right\}
$$

hence the set is Borel measurable.

THEOREM 2.1. If the limit of the sequence (2.1) is 0 , then $N(x(t))=\infty$ for almost all $t$ (in the sense of Borel measure).

Proof. Fomula (1.4) and Lemma 1.1 imply that

$$
\sum_{k=1}^{2^{n}} \phi_{n k}(x(t))=\phi(x(t))>0
$$

for almost all $t$, for every $n$.

\section{Observe}

$$
\begin{aligned}
& \int_{-\infty}^{\infty} \liminf _{n \rightarrow \infty} \max \phi_{n k}^{2}(x) d x \leqq \lim _{n \rightarrow \infty} \int_{-\infty}^{\infty} \max \phi_{n k}^{2}(x) d x \quad \text { (Fatou's lemma) } \\
& \leqq \lim _{n \rightarrow \infty} \int_{-\infty}^{\infty} \sum_{k=1}^{2^{n}} \phi_{n k}^{2}(x) d x=\lim _{n \rightarrow \infty} \frac{1}{2 \pi} \sum_{k=1}^{2^{n}} \int_{-\infty}^{\infty}\left|f_{n k}(u)\right|^{2} d u \\
& =0 \quad \text { (by hypothesis); }
\end{aligned}
$$

therefore, for almost all $x, \lim \inf _{n \rightarrow \infty} \max _{k} \phi_{n k}(x)=0$. This implies

$$
\liminf _{n \rightarrow \infty} \max _{k} \phi_{n k}(x(t))=0
$$

for almost all $t$ because a function with a local time maps sets of positive measure into similar sets. The relations (2.3) and (2.4) together imply that

$$
\lim _{n \rightarrow \infty} N_{n}(x(t))=\infty, \text { for almost all } t .
$$

This and Lemmas 2.3 and 2.4 imply the assertion of the theorem. 
Corollary 2.1. Under the hypothesis of Theorem 2.1, the set $\{y: N(y)<\infty\}$ is of $\nu$-measure 0 ; in other words, $x(\cdot)$ spends no time in this set.

Proof. Lemmas 2.3 and 2.4 imply

$$
\nu\{y: N(y)<\infty\} \leqq \nu\left\{y: \lim _{n \rightarrow \infty} N_{n}(y)<\infty\right\}
$$

and the definition of $\nu$, and Theorem 2.1 imply

$$
\nu\left\{y: \lim _{n \rightarrow \infty} N_{n}(y)<\infty\right\}=\mu\left\{t: \lim _{n \rightarrow \infty} N_{n}(x(t))<\infty\right\}=0 .
$$

\section{An inequality for the range of values of a function.}

Lemma 3.1. Let $x$ be a Borel function on $(I, \mathscr{B}, \mu)$; put

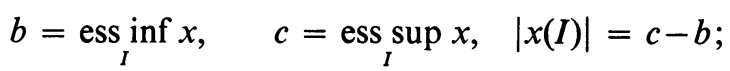

then, for every $\varepsilon, 0 \leqq \varepsilon<1$, and nonnegative integer $m$,

$$
|x(I)|^{2 m+1+\varepsilon} \geqq \mu^{2}(I) \cdot K / \int_{-\infty}^{\infty}|u|^{2 m+\varepsilon}|f(u)|^{2} d u,
$$

where $K$ is a numerical constant. If the integral in the denominator is infinite, the fraction is understood to be 0 ; in this case, the inequality is trivial.

Proof. If either $|b|$ or $c$ is infinite, (3.1) is obvious; thus, we now assume both to be finite. We have

$$
\mu(I)=\int_{-\infty}^{\infty} \frac{e^{-i u c}-e^{-i u b}}{-2 \pi i u} f(u) d u ;
$$

for, on one hand, $\mu(I)$ is equal to

$$
\int_{b}^{c} \phi(x) d x
$$

because the integral of $\phi$ over the complement of the range of $x(t), t \in I$, vanishes; on the other hand, by Parseval's theorem the integrals in (3.2) and (3.3) are equal.

First we prove (3.1) for $m=0$. The integral in (3.2) is dominated by

$$
\int_{-\infty}^{\infty}|u|^{-\varepsilon / 2}\left|\frac{e^{-i u c}-e^{-i u b}}{2 \pi u}\right||u|^{\varepsilon / 2}|f(u)| d u,
$$

whose square, by the Cauchy-Schwarz inequality, is dominated by

$$
\begin{aligned}
\int_{-\infty}^{\infty}|u|^{-\varepsilon}\left|\frac{1-e^{-i u(c-b)}}{2 \pi u}\right|^{2} d u \cdot \int_{-\infty}^{\infty}|u|^{\varepsilon}|f(u)|^{2} d u \\
=\text { constant } \cdot(c-b)^{1+\varepsilon} \int_{-\infty}^{\infty}|u|^{\varepsilon}|f(u)|^{2} d u .
\end{aligned}
$$

Now suppose $m \geqq 1$. We assume that the integral in (3.1) is finite; otherwise, there is nothing to prove. The finiteness of this integral implies that $\phi$ and its first $m$ derivatives $\phi^{(1)}, \ldots, \phi^{(m)}$ exist and are square integrable [6, p. 92]; in particular, $\phi$ and its first $m-1$ derivatives are continuous; thus, they necessarily vanish at $b$ 
and $c$ because they vanish everywhere outside $[b, c]$. Using this fact we find, by successive integration by parts:

$$
\int_{b}^{c} \phi(x) d x=\frac{(-1)^{m}}{m !} \int_{b}^{c}(x-b)^{m} \phi^{(m)}(x) d x .
$$

Apply the Parseval relation to the integral on the right-hand side. The Fourier transform of $\phi^{(m)}$ is $(i u)^{m} f(u)$, and that of the function

$$
\begin{aligned}
g(x) & =(x-b)^{m}, & & b \leqq x \leqq c, \\
& =0, & & \text { elsewhere }
\end{aligned}
$$

is

$$
\int_{b}^{c} e^{i u x}(x-b)^{m} d x
$$

hence, the integral on the right-hand side of (3.4) is equal to

$$
\frac{(-i)^{m}}{2 \pi m !} \int_{-\infty}^{\infty}\left(\int_{b}^{c} e^{i u x}(x-b)^{m} d x\right)\left(u^{m} f(u)\right) d u .
$$

Multiply the first function in the integrand by $|u|^{-\varepsilon / 2}$, the second by $|u|^{\varepsilon / 2}$, and apply the Cauchy-Schwarz inequality: the square of the integral is dominated by the product of the two integrals

$$
\int_{\infty}^{\infty}|u|^{2 m+\varepsilon}|f(u)|^{2} d u
$$

and

$$
\frac{1}{(2 \pi m !)^{2}} \int_{-\infty}^{\infty}|u|^{-\varepsilon}\left|\int_{b}^{c} e^{i u x}(x-b)^{m} d x\right|^{2} d u .
$$

The latter integral is dominated by a constant multiple of $(c-b)^{2 m+1+\varepsilon}$; this can be verified by successive substitution of variables in the inner and outer integrals: $y=(x-b) /(c-b)$ and $v=u(c-b)$. The proof of the inequality (3.1) is now complete: compare the estimate of the right-hand side of (3.4) to the left-hand side; the latter, as shown in the remark leading to (3.3), is equal to $\mu(I)$.

4. Absence of Hölder conditions and infinite $\gamma$-variation for functions with a smooth local time. We apply the inequality (3.1) in the particular case in which $I$ is the closed unit interval and $\mu$ the usual Borel measure. We continue to use the notation of $\S 2$; here $\mu\left(I_{n k}\right)=2^{-n}, k=1, \ldots, 2^{n}$. Put $I_{n k}$ and $f_{n k}$ in the places of $I$ and $f$, respectively, in (3.1) for $k=1, \ldots, 2^{n}$; and let $x$ be a Borel function. Two inequalities follow:

$$
\begin{gathered}
\min _{k}\left|x\left(I_{n k}\right)\right|^{2 m+1+\varepsilon} \geqq K / 2^{2 n} \sum_{k} \int_{-\infty}^{\infty}|u|^{2 m+\varepsilon}\left|f_{n k}(u)\right|^{2} d u \\
\sum_{k}\left|x\left(I_{n k}\right)\right|^{2 m+1+\varepsilon} \geqq K / \sum_{k} \int_{-\infty}^{\infty}|u|^{2 m+\varepsilon}\left|f_{n k}(u)\right|^{2} d u
\end{gathered}
$$


The inequality (4.1) is an elementary algebraic consequence of the $2^{n}$ inequalities (3.1); and (4.2) is obtained by averaging over the $2^{n}$ inequalities (3.1), and noting that the arithmetic mean is at least equal to the harmonic mean.

For $\gamma \geqq 1$, the $\gamma$-variation of the function $x(t), 0 \leqq t \leqq 1$, is defined as

$$
\lim _{n \rightarrow \infty} \sup _{0 \leqq t_{1} \leqq \cdots \leqq t_{n} \leqq 1} \sum_{j=1}^{n-1}\left|x\left(t_{j+1}\right)-x\left(t_{j}\right)\right|^{\gamma} .
$$

An immediate implication of the inequality (4.2) is

LEMMA 4.1. If the $\lim \inf (n \rightarrow \infty)$ of the denominator in (4.2) is equal to 0 , then the $\gamma$-variation of $x$ is infinite for $\gamma=2 m+1+\varepsilon$.

The function $x$ is said to satisfy a Hölder condition of order $p$ at the point $t_{0}$ in $[0,1]$ if there are positive constants $h$ and $\delta$ such that $\left|x(t)-x\left(t_{0}\right)\right| \leqq h\left|t-t_{0}\right|^{p}$ whenever $\left|t-t_{0}\right| \leqq \delta$.

LEMMA 4.2. Let $x$ satisfy a Hölder condition of order $p$ at the point $t_{0}$ for some constants $h$ and $\delta$. If $I$ is a subinterval of $[0,1]$ of positive length $|I| \leqq \delta$, and I contains $t_{0}$, then

$$
|x(I)| \leqq 2|I|^{p} h .
$$

Proof. In order to prove the lemma we shall show that the inequality

$$
|x(t)-x(s)| \leqq 2|I|^{p} h
$$

is satisfied for all points $s$ and $t$ in $I$. For $s=t$ the inequality is trivial. If $s \neq t$ and $t=t_{0}$ (or $s=t_{0}$ ), then (4.4) is a consequence of the assumed Hölder condition. If $s, t$ and $t_{0}$ are distinct, we deduce (4.4) by noting that (triangle inequality)

$$
\frac{|x(t)-x(s)|}{|I|^{p}} \leqq \frac{\left|x(t)-x\left(t_{0}\right)\right|}{\left|t-t_{0}\right|^{p}} \frac{\left|t-t_{0}\right|^{p}}{|I|^{p}}+\frac{\left|x(s)-x\left(t_{0}\right)\right|}{\left|s-t_{0}\right|^{p}} \frac{\left|s-t_{0}\right|^{p}}{|I|^{p}}
$$

and that $\left|t-t_{0}\right| \leqq|I|,\left|s-t_{0}\right| \leqq|I|$, and then invoking the assumed Hölder condition.

Corollary 4.1. If

$$
\limsup _{n \rightarrow \infty} 2^{n p} \min _{k}\left|x\left(I_{n k}\right)\right|=\infty,
$$

then $x$ does not satisfy a Hölder condition of order $p$ at any point of $[0,1]$.

Combining Corollary 4.1 and the inequality (4.1) we conclude:

LEMMA 4.3. If the $\lim \inf (n \rightarrow \infty)$ of the denominator in (4.1) is equal to 0 , then $x$ nowhere satisfies a Hölder condition of order $p=2 /(2 m+1+\varepsilon)$; in particular, if the hypothesis holds for $m=1$, then $x$ is nowhere differentiable.

5. Local times and sample functions of Gaussian processes. Let $(I, \mathscr{B}, \mu)$ be as defined in the introduction; $(\Omega, \mathscr{F}, P)$ a probability space; and $X=X(t, \omega)$ a real 
valued stochastic process on $\Omega$, measurable with respect to $\mathscr{B} \times \mathscr{F}$. For each sample function $X(\cdot, \omega)$ we define the occupation time distribution: $\nu(A, \omega)$ $=\mu\left(X^{-1}(A)\right)$, for every linear Borel set $A$. The Fourier-Stieltjes transform of the corresponding distribution function is, by formula (1.2), given by

$$
f(u, \omega)=\int_{I} e^{i u X(t, \omega)} d \mu(t), \quad-\infty<u<\infty ;
$$

it is a measurable function of $(u, \omega)$.

Let $E$ be the expectation operator corresponding to the probability measure $P$. When a function of $(t, \omega)$ or $(u, \omega)$ appears under the expectation operator, the argument $\omega$ will be suppressed; for example, $E f(u, \omega)$ is written $E f(u)$.

In this section $X$ is a Gaussian process. It will be assumed that $E X(t) \equiv 0(t \in I)$. The application of the above theory of local times to Gaussian processes depends almost entirely on this formula:

$$
\begin{aligned}
& E \int_{-\infty}^{\infty}|u|^{p}|f(u)|^{2} d u \\
& =2^{(p+1) / 2} \Gamma\left(\frac{p+1}{2}\right)(2 \pi)^{-1} \int_{I} \int_{I}\left[E(X(s)-X(t))^{2}\right]^{-(p+1) / 2} d \mu(s) d \mu(t), \quad p \geqq 0 .
\end{aligned}
$$

The proof is: $X(t, \omega)$ and $f(u, \omega)$ are measurable functions of their respective pairs of arguments; thus, application of Fubini's theorem permits the interchange of order of integration:

$$
\begin{aligned}
E \int_{-\infty}^{\infty}|u|^{p}|f(u)|^{2} d u & =\int_{-\infty}^{\infty}|u|^{p} \int_{I} \int_{I} E e^{i u(X(t)-X(s))} d \mu(s) d \mu(t) d u \\
& =\int_{-\infty}^{\infty}|u|^{p} \int_{I} \int_{I} \exp \left[-\frac{1}{2} u^{2} E(X(t)-X(s))^{2}\right] d \mu(s) d \mu(t) d u .
\end{aligned}
$$

Another interchange of order of integration, and the change of variable $v^{2}=$ $u^{2} E(X(t)-X(s))^{2}$ show that the last integral is equal to that on the right-hand side of (5.1).

For use in $\S 6$ we record a second formula:

$$
\begin{aligned}
& E \int_{-\infty}^{\infty} e^{u b}|f(u)|^{2} d u \\
& =\int_{I} \int_{I}(2 \pi)^{1 / 2}\left[E(X(t)-X(s))^{2}\right]^{-1 / 2} \exp \left[b^{2} / 2 E(X(t)-X(s))^{2}\right] d \mu(s) d \mu(t),
\end{aligned}
$$

for any real $b$. (The proof is similar to that for (5.1) with the modification that the formula for the moment generating function of the Gaussian distribution is used.)

LEMMA 5.1. If the integral on the right-hand side of (5.1) is finite for some $p \geqq 0$, then, for almost all $\omega, \nu(\cdot, \omega)$ is absolutely continuous with respect to linear Borel measure. Its derivative, the local time of $X(\cdot, \omega)$, is square integrable. The first $[p / 2]$ derivatives of the local time exist and are square integrable $([u]=$ integral part of $u)$. 
Proof. The hypothesis implies: for almost all $\omega,|u|^{[p / 2]}|f(u, \omega)|$ is square integrable; thus, the conclusion follows the application of $[6, p .92]$.

We now take $I$ to be unit interval with the usual Borel measure, and return to the notation of the previous section.

LEMMA 5.2. If the integral on the right-hand side of equation (5.1) is finite, then for almost all $\omega$,

$$
\liminf _{n \rightarrow \infty} \sum_{k} \int_{-\infty}^{\infty}|u|^{p}\left|f_{n k}(u, \omega)\right|^{2} d u=0 .
$$

Proof. The union of the squares $I_{n k} \times I_{n k}, k=1, \ldots, 2^{n}$ forms a decreasing sequence of Borel sets $(n=1,2, \ldots)$ of measure tending to 0 : therefore,

$$
\lim _{n \rightarrow \infty} \sum_{k} \int_{I_{n k}} \int_{I_{n k}}[E(X(s)-X(t))]^{-(p+1) / 2} d \mu(s) d \mu(t)=0 ;
$$

thus, by application of (5.1),

$$
\lim _{n \rightarrow \infty} E \sum_{k} \int_{-\infty}^{\infty}|u|^{p}\left|f_{n k}(u)\right|^{2} d u=0,
$$

and Fatou's lemma completes the proof.

THEOREM 5.1. Let $X$ be a Gaussian process on $[0,1]$. Let $m$ be a nonnegative integer, $\varepsilon$ a real number such that $0 \leqq \varepsilon<1$, and $p$ defined as $p=2 m+\varepsilon$. If the integral on the right-hand side of (5.1) is finite, then, for almost all $\omega$ :

(a) The $\gamma$-variation of $X(\cdot, \omega)$ is infinite for $\gamma=p+1$;

(b) $X(\cdot, \omega)$ nowhere satisfies a Hölder condition of order $2 /(p+1)$;

(c) $N(X(t, \omega))$ is infinite for almost all $t$ (Theorem 2.1). This is valid as long as the hypothesis is fulfilled for $p=0$.

Proof. The assertion (a) is based on Lemmas 4.1 and 5.2; (b) on Lemmas 4.3 and 5.2; and (c) on Theorem 2.1 and Lemma 5.2 (for $p=0$ ).

Suppose that there are constants $C>0$ and $\beta>0$ such that

$$
E(X(s)-X(t))^{2} \geqq C|t-s|^{\beta}, \quad 0 \leqq s, t \leqq 1 ;
$$

then the integral (5.1) is finite if $\beta(p+1)<2$. If $\beta<2$, then (5.1) is finite at least for $p=0$; therefore, conclusion (c) of Theorem 5.1 holds. If for some $\varepsilon, 0 \leqq \varepsilon<1$, we have $\beta<2 /(1+\varepsilon)$, then, for $\gamma=1+\varepsilon$ the $\gamma$-variation of $X(\cdot, \omega)$ is infinite for almost all $\omega$; this follows from assertion (a) with $m=0$. Assertion (b) implies: if

$$
\beta<2 /(2 m+\varepsilon+1)
$$

then $X(\cdot, \omega)$ nowhere satisfies a Hölder condition of order $2 /(2 m+\varepsilon+1)$.

In order to prove the nowhere differentiability of $X(\cdot, \omega)$, we shall show that the sample function nowhere satisfies a Hölder condition of order 1 . The result on 
Hölder conditions just given is not completely satisfactory because 1 is not expressible in the form $2 /(2 m+\varepsilon+1)$ since $\varepsilon<1$. The best result available from (5.4) is: if $\beta<2 / 3(m=1, \varepsilon=0)$, there is nowhere a Hölder condition of order $2 / 3$, so that the sample function is nowhere differentiable. This will now be improved.

THEOREM 5.2. The sample functions are nowhere differentiable if (5.3) holds for some $\beta<1$.

Proof. Let $\varepsilon$ be a real number satisfying the double inequality

$$
\beta /(2-\beta)<\varepsilon<1 ;
$$

such a number exists under the assumption $\beta<1$. Put $m=0$ in the inequality (4.1) and multiply both sides by $2^{n(1+\varepsilon)}$ :

$$
2^{n(1+\varepsilon)} \min \left|x\left(I_{n k}\right)\right|^{1+\varepsilon} \geqq K / 2^{n(1-\varepsilon)} \sum_{k} \int_{-\infty}^{\infty}|u|^{\varepsilon}\left|f_{n k}(u, \omega)\right|^{2} d u .
$$

Under the hypothesis (5.3) and by the formula (5.1) the expected value of the denominator in (5.6) is bounded above by a constant multiple of

$$
2^{n(2-\varepsilon)} \int_{0}^{2^{-n}} \int_{0}^{2^{-n}}|t-s|^{-\beta(\varepsilon+1) / 2} d s d t=2^{n[\beta(\varepsilon+1) / 2-\varepsilon]} \int_{0}^{1} \int_{0}^{1}|t-s|^{-\beta(\varepsilon+1) / 2} d s d t .
$$

The latter double integral is finite because $\varepsilon$ and $\beta$ are less than 1; and the factor multiplying the integral converges to 0 as $n \rightarrow \infty$, under the assumption (5.5); thus (Fatou's lemma), the $\lim \inf (n \rightarrow \infty)$ of the denominator in (5.6) is 0 , for almost all $\omega$; hence, by Corollary $4.1(p=1), X(\cdot, \omega)$ nowhere satisfies a Hölder condition of order 1 .

6. Analytic local time: an application to Brownian motion over Hilbert space. We return to the general space $I$ and recall some results given in [2] for the case of the unit interval. If $\phi$ is analytic, then $x$ spends positive time in every Borel set of positive measure; therefore, $x$ is unbounded. A sufficient condition for the analyticity of $\phi$ is that there exist $b>0$ such that

$$
\int_{-\infty}^{\infty} e^{u b}|f(u)|^{2} d u<\infty
$$

thus, if there exists a positive Borel measure on $\mathscr{B}$ for which (6.1) holds, then $x$ is unbounded on $I$.

Now let $X(t, \omega), t \in I$ be a measurable Gaussian process defined as in $\S 5$.

LEMMA 6.1. If there exists a positive Borel measure $\mu$ on I, and $b>0$ such that

$$
\int_{I} \int_{I} \exp \left[b^{2} / E(X(t)-X(s))^{2}\right] d \mu(s) d \mu(t)<\infty
$$

then, for almost all $\omega, X(\cdot, \omega)$ is unbounded on $I$. 
Proof. The hypothesis implies that

$$
\int_{I} \int_{I}\left[E(X(s)-X(t))^{2}\right]^{-1} d \mu(s) d \mu(t)<\infty
$$

because $u^{-1}$ tends to infinity more slowly than $\exp \left(b^{2} / u\right)$ for $u \downarrow 0$; thus, applying the Cauchy-Schwarz inequality to the integral on the right-hand side of (5.2), we find the left-hand side to be finite; consequently, for almost all $\omega,(6.1)$ holds with $f=f(u, \omega)$.

Take $I$ to be the space $l_{2}$ of all real square summable sequences $t=\left(t_{n}\right)$ with the usual norm $\|\cdot\|$ defined by $\|t\|^{2}=\sum_{n} t_{n}^{2}$. Let $X$ be a measurable version of Levy's Brownian motion, a Gaussian process such that

$$
E X(t) \equiv 0, \quad E X^{2}(0)=0, \quad E(X(t)-X(s))^{2}=\|t-s\| .
$$

In [1] it is shown that on each compact subset of $l_{2}$ either the sample functions are continuous, or if not, there is a point in the subset at which each sample function is discontinuous. In the particular case of a compact ellipsoid sufficient conditions for continuity were given; now Lemma 6.1 will be used to get sufficient conditions for the unboundedness of the sample functions. The method can also be used for other compact sets in $l_{2}$; for example, a cube.

An ellipsoid is a set of the form

$$
\left\{t: \sum_{n} t_{n}^{2} / \lambda_{n}^{2} \leqq 1\right\}
$$

where $\left\{\lambda_{n}\right\}$ is a sequence of real numbers. The ellipsoid is compact if $\lambda_{n} \rightarrow 0$ $[3$, p. 28].

THEOREM 6.1. Let $S$ be a compact ellipsoid in $l_{2}$, and $\left(\lambda_{n}\right)$ the sequence in (6.3). If for some $p, 1<p<2$,

$$
\sum_{n} \frac{1}{n^{p} \lambda_{n}^{2}}<\infty
$$

then the sample functions are unbounded over $S$.

(The goal of the proof is the construction of a positive Borel measure on $S$ for which (6.2) holds.)

Proof. Let $X_{1}, X_{2}, \ldots$ be a sequence of independent random variables on some probability space having Gaussian distributions with expectations 0 and $E X_{n}^{2}=n^{-p}$. For each integer $k>0$ and real numbers $t_{1}, \ldots, t_{k}$ define the measure of the cylinder set $\left\{s: s_{1} \leqq t_{1}, \ldots, s_{k} \leqq t_{k}\right\}$ in $l_{2}$ as $\operatorname{Pr}\left(X_{1} \leqq t_{1}, \ldots, X_{k} \leqq t_{k}\right)$. This cylinder set measure is extendable to a countably additive measure (denoted also by $\mu$ ) on the Borel sets of $l_{2}$; indeed, the series $\sum_{n} X_{n}^{2}$ converges with probability 1 because the sequence of variances converges (cf. [3, p. 334, Theorem 7]). 
The condition (6.4) also implies that the series $\sum_{n} X_{n}^{2} / \lambda_{n}^{2}$ converges with probability 1 ; therefore, for every $n \geqq 1$, the series may be represented as the sum of the two independent sub-sums

$$
\sum_{j=1}^{n} X_{j}^{2} / \lambda_{j}^{2} \text { and } \sum_{j=n+1}^{\infty} X_{j}^{2} / \lambda_{j}^{2}
$$

This representation implies

$$
\operatorname{Pr}\left(\sum_{n} X_{n}^{2} / \lambda_{n}^{2} \leqq 1\right)>0 ;
$$

indeed, for every $n$, the distribution of the first sub-sum certainly assigns positive probability to the interval of values $\left[0, \frac{1}{2}\right]$, and, if $n$ is sufficiently large, so does the distribution of the second sub-sum; thus the convolution of the distributions assigns positive probability to $[0,1]$; hence, $\mu(S)>0$.

The next step in the proof is the estimation of the double integral in (6.2) when $\mu$ is the measure just constructed and $E(X(t)-X(s))^{2}=\|t-s\|$. Let $\left\{Y_{n}\right\}$ be a sequence of random variables on the same space as $\left\{X_{n}\right\}$, distributed identically as the latter but independent of it. By definition of $\mu$, the double integral in (6.2) is equal to

$$
E\left\{\exp \left[b^{2} /\left(\sum_{n}\left(X_{n}-Y_{n}\right)^{2}\right)^{1 / 2}\right]\right\}
$$

thus, since $X_{n}-Y_{n}$ is distributed as $\sqrt{ } 2 X_{n}$ :

$$
\int_{l_{2}} \int_{l_{2}} \exp \left(b^{2} / E(X(s)-X(t))^{2}\right) d \mu(s) d \mu(t)=E\left\{\exp \left[b^{2} / \sqrt{ } 2\left(\sum_{n} X_{n}^{2}\right)^{1 / 2}\right]\right\}
$$

Define

$$
\begin{aligned}
U_{n}=1 & \text { if }\left|X_{n}\right|>n^{-p / 2}, \\
=0 & \text { if }\left|X_{n}\right| \leqq n^{-p / 2} .
\end{aligned}
$$

The $U$ 's are independent and have a common Bernoulli distribution with mean $\operatorname{Pr}\left(\left|X_{n}\right|>n^{-p / 2}\right)=0.3174$ (approximately); and, from (6.6):

$$
E\left\{\exp \left[b^{2} / \sqrt{ } 2\left(\sum_{n} X_{n}^{2}\right)^{1 / 2}\right]\right\} \leqq E\left\{\exp \left[b^{2} / \sqrt{ } 2\left(\sum_{n} n^{-p} U_{n}\right)^{1 / 2}\right]\right\}
$$

The final step in the proof is showing that the latter expectation is finite. Recall that the expected value of a nonnegative random variable $Z$ is finite if

$$
\int_{0}^{\infty} \operatorname{Pr}(Z>t) d t<\infty
$$

thus, the expectation in (6.7) is finite if

$$
\int_{1}^{\infty} \operatorname{Pr}\left(\sum n^{-p} U_{n}<b^{4} / 2|\log t|^{2}\right) d t<\infty
$$

For every $\tau>0$ the inequality $\sum_{n} n^{-p} U_{n}<\tau$ holds only if the terms up to index 
$n>\tau^{-1 / p}$ are all equal to 0 ; thus, by the independence of the $\left\{U_{n}\right\}$ and common distribution:

$$
\begin{aligned}
\operatorname{Pr}\left(\sum n^{-p} U_{n}<\tau\right) & \leqq \operatorname{Pr}\left(U_{j}=0, j=1, \ldots,\left[\tau^{-1 / p}\right]\right) \\
& \leqq\left(\operatorname{Pr}\left(U_{1}=0\right)\right)^{[\tau-1 / p]} \leqq(0.7)^{[\tau-1 / p]} ;
\end{aligned}
$$

therefore, putting $\tau=b^{4} / 2|\log t|^{2}$, we see that the integral in (6.8) is at most

$$
\int^{\infty}(1 / 0.7) \exp \left\{2^{1 / p}|\log t|^{2 / p} b^{-4 / p} \log (0.7)\right\} d t ;
$$

it is finite because $p<2$.

The measure $\mu$ assigns positive measure to $S$, and satisfies (6.2); hence, the sample functions are unbounded on $S$.

EXAMPLE. Suppose $S$ is the ellipsoid (6.3) and $\lambda_{n}=n^{-r}$, where $r>0$. The sample functions are continuous on $S$ if $r>1$; indeed, in this case $\sum_{n}\left|\lambda_{n}\right|^{p}<\infty$ for some $p<1$ and the continuity condition given in [1] is satisfied. The sample functions are unbounded on $S$ if $r<1 / 2$; in fact, there exist number $p$ and $\varepsilon, 1<p<2, \varepsilon>0$, such that $1<p<2$ and $2 r<(p-1)-\varepsilon$, and the series $\sum_{n} n^{-p} n^{2 r}$ converges.

Added in Proof. S. Varadhan showed that Theorem 6.1 is valid also for $1<p \leqq 3$. From the inequality

$$
\prod_{n \geqq 1}\left(1+\lambda n^{-p}\right)^{-1} \leqq \exp \left(-c \lambda^{1 / p}\right), \quad c>0 \text { constant }
$$

the formula for the moment generating function of $\sum_{n \geqq 1} X_{n}^{2}$, and a Chebyshev-type inequality, we conclude that

$$
P\left[\sum X_{n}^{2} \leqq t\right] \leqq \exp \left[- \text { constant } / t^{1 /(p-1)}\right], \quad t>0 .
$$

This implies the finiteness of (6.5). In the example $\lambda_{n}=n^{-r}$ the sample functions are unbounded if $r<1$.

\section{REFERENCES}

1. S. M. Berman, Some continuity properties of Brownian motion with the time parameter in Hilbert space, Trans. Amer. Math. Soc. 131 (1968), 182-198.

2. - Local times and sample function properties of stationary Gaussian processes, Trans. Amer. Math. Soc. 137 (1969), 277-300.

3. I. M. Gelfand and N. Ya. Vilenkin, Generalized functions. Vol. 4, Applications of harmonic analysis, English transl., Academic Press, New York, 1964.

4. M. B. Marcus, Hölder conditions for Gaussian processes with stationary increments, Trans. Amer. Math. Soc. 134 (1968), 29-52.

5. D. Slepian, The one-sided barrier problem for Gaussian noise, Bell System Tech. J. 41 (1962), 463-501.

6. E. C. Titchmarsh, Introduction to the theory of Fourier integrals, Clarendon Press, Oxford, 1937.

7. J. Yeh, Differentiability of sample functions in Gaussian processes, Proc. Amer. Math. Soc. 18 (1967), 105-108; correction 19 (1968), 843.

Courant Institute of Mathematical Sciences, New York University, New York, New York 